give an idea of the scope. The photograph herewith shows the ring system when a residual aberration, in the sense of "over-correction," of $0.5 \lambda$ is present at the best focus, and when the reference plane is taken sufficiently far outside the focus to make the pathdifference $d p=\mathrm{r} \cdot 8 \lambda$. The small central concentration and the persistent individuality of the interference rings are shown. The first "broad" diffraction ring is now formed through the low intensity of the first bright interference ring. The whole of the above work relates to nearly monochromatic light only.

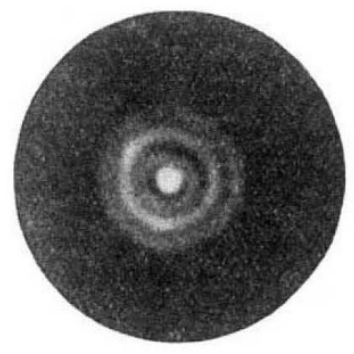

A good many of the broad features of these aberration and "out of focus" effects can be explained without difficulty on the basis of the Fresnel zone theory, and it is hoped to include a short discussion of this kind in the paper. No more need be said at present except to direct the attention of the readers of Nature to the importance of the paper on "Star Discs" to which the reference is given.

\section{C. Martin.}

Imperial College of Science and Technology, November 25.

\section{"Phenomena of Materialisation."}

IN NATURE of November I8 I find what purports to be a review of Dr. von Schrenck-Notzing's work translated by me under the above title. I have always supposed that a reviewer should tell the reader what the book is about. The review in question is headed "The Newer Spiritualism," and begins: " Of making many books ' on spiritualism "there is no end." It states that the phenomena are alleged to have "a spiritistic interpretation," and refers to "the numerous photographs of her [the medium] sandwiched between faked spirit photographs." All this is thoroughly misleading. The book is not about spiritualism. Both the author (p. 30) and the translator (p. x) discard the "spirit hypothesis" as unnecessary. The author says that "it impedes and hinders in every way serious scientific investigation." The book contains no portrait of the medium, and not a single "spirit photograph," faked or otherwise. The photographs reproduced have not been manipulated in any way except Nos. 127, I28, 134, 136, 138, and 140 , in which, for purposes of publication, the sex characteristics have been obliterated. These six photographs are marked "retouched," and the reason for retouching is stated in the text.

The review abounds with other inaccuracies. A red light was not always employed (see p. 306). Mme. Bisson did not "hop in and out" of the cabinet. The rare occasions on which she entered it are stated, as are all the other conditions, with what the reviewer calls "dreary uniformitv," but with what the ordinary scientific reader would call conscientious accuracy.

The reviewer's challenge to "exhibit" the phenomena in London before well-known hostile critics is about as reasonable as to ask a performing mouse to No. 2667 , VOL. IO6] exhibit its tricks before an audience of hungry cats. The psychological element would probably produce similar inhibitions in both cases. The phenomena are nearly as rare as the fall of a meteorite from the sky, and nearly as spontaneous as the production of biological "sports." They cannot, therefore, be produced before a massed audience. It is useless to think that any living individual is eminent enough to carry a general conviction of the reality of the phenomena, even though he be personally convinced. The only resource is to take the phenomena as and when they come, to record them carefully, if possible by photography and other instrumental means, and to make the experimental conditions gradually more rigid until we can only conclude that we are face to face with a new set of biological phenomena exhibiting the known powers of the human organism in an intensified and much accelerated form. And this is precisely what Dr. von Schrenck-Notzing has done.

$$
\text { E. E. Fournier D'Albe. }
$$

It will be well to deal with Dr. Fournier d'Albe's charges against my accuracy seriatim:

(I) "Both the author (p. 3o) and the translator (p. $x$ ) discard the "spirit hypothesis' as unnecessary." I might have made this clearer, but the repudiation is not easy to reconcile with the contents of a book crammed with references to occult phenomena, as, e.g., mediumistic, psychic, and telekinetic.

(2) "The book contains no portraits of the medium, and not a single 'spirit photograph.'" This is an amazing statement. There are 225 illustrations. Of these there are 13 "drawings," so-called; all the rest are flashlight photographs of the medium (if not, then of whom?) in various attitudes, a large number showing the teleplasma issuing from her mouth, etc. There are 20 flashlight photographs-" "phantasms" they are called-of dead and living people. Among the former Mme. Bisson recognised the features of a deceased nephew, Georges Thurner, and also of her husband, who died in IgI2.

(3) "A red light was not always employed (see p. 306)." "All the sittings took place in a red light, so that during the four years there was not a single dark séance" (p. 2I). The translator may be left to reconcile this statement with the exception to which he gives the reference.

(4) "Mme. Bisson did not 'hop in and out' of the cabinet." Probably she neither hopped nor skipped, but her visits to the cabinet were frequent enough to arouse suspicion as to collusion with a medium over whom she had "absolute control" (p. 59). Dr. Fournier d'Albe does not appear to have been present at the sittings.

(5) "The reviewer's challenge" cannot be accepted because the phenomena cannot "be produced before a mixed audience. . . ." "We are face to face with a new set of biological phenomena." So long as those who assert their belief in teleplastic exudations from the body and in the genuineness of photographs of the dead refuse to submit these "new biological" phenomena to the conjoint judgment of men of science and conjurers, they must not be surprised that their socalled "evidence" carries no weight save among the credulous.

The Reviewkr.

\section{Higher Forestry Education for the Empire.}

A CORRESPONDENT has sent us some remarks upon Prof. Stebbing's letter dealing with forestry education in Nature of December 2, but he has omitted to give his full name and address. No use can, therefore, be made of his communication.-EDIToR, NATURE. 